\title{
Exploring the Surface of Pb-doped Manganese Perovskite
}

\author{
C.N. Borca, and P.A. Dowben
}

Physics Department, University of Nebraska at Lincoln, Lincoln, NE 68588

Recently, there have been a surge of interest in transition metal oxides due to their unique physical properties, e.g. colossal magnetoresistance (CMR) [1] and extremely high degree of spin polarization [2]. Systems with $100 \%$ spin-polarization (or half-metals) are desirable in spin-valves, spin-tunnel junctions and other spin dependent devices and structures. In the present study, we investigated the importance of the surface and near-surface composition on the value of spin-asymmetry in $\mathrm{La}_{0.65} \mathrm{~Pb}_{0.35} \mathrm{MnO}_{3}$ thin films.

The $\mathrm{La}_{0.65} \mathrm{~Pb}_{0.35} \mathrm{MnO}_{3}$ thin films (1000 $\AA$ in thickness) were grown on (100) $\mathrm{LaAlO}_{3}$ substrates by $\mathrm{RF}$ sputtering [3]. The films are polycrystalline and, highly oriented along the substrate normal direction and single phase. For the detailed studies of the surface composition, the films were installed into an ultra high vacuum system (base pressure $2 \times 10^{-10}$ torr). To ensure surface cleanliness, the "asdeposited" samples were gently annealed to $250^{\circ} \mathrm{C}$ for $10-14$ hours, in oxygen atmosphere of $1 \times 10^{-6}$ torr. After careful analysis of this initial surface, the samples were further annealed in vacuum to $520^{\circ} \mathrm{C}$ for $10-14$ hours. This second thermal treatment produced a heavily annealed surface with completely different composition and structure, as discussed below.

Changes in surface topography were obtained using a scanning probe microscope (STM), as seenin Figure 1. Panel 1(a) corresponds to the "as-deposited" sample, with a roughness of $5 \mathrm{~nm}$, which indicates a smooth surface, with atomically flat terraces. The heavily annealed surface shown in Figure 1(b) suggests the existence of mixed phases and an increased surface roughness of $50 \mathrm{~nm}$. The changes induced into the surface composition and structure due to heavy annealing have been confirmed by low energy electron diffraction (LEED) and X-ray photoemission spectroscopy (XPS). LEED shows the appearance of sharp superlattice spots in the case of the heavily annealed surfaces, indicating a possible four-fold modulation in the Mn-O planes. XPS of the $\mathrm{La}, \mathrm{Pb}$ and Mn core levels, acquired as a function of photoelectron emission angles, show that the heavily anneded surface goes through a restructuring transition characterized by the formation of a Ruddlesden-Popper phase ( $\mathrm{La}_{1-}$ $\left.{ }_{\mathrm{x}} \mathrm{Pb}_{\mathrm{x}}\right) \mathrm{MnO}_{4}$ with a possibly embedded $\mathrm{MnO}$ columnar phase.

The effect of surface composition on the spin-resolved band structure of this material can be seen using inverse photoemission spectroscopy. Figure 2 shows the spin-resolved spectra of the unoccupied band structure of the as-deposited surface (a) and of the heavily annealed surface (b). The value of the surface spin-asymmetry is obtained by taking the ratio between the difference and the sum of the spin-up and spin-down spectra for each surface, and it is shown at the bottom of panels (a) and (b) in Figure 2. The spin-asymmetry at room temperature for the as-deposited surface reaches $80 \%$ above background, while the heavily annealed surface shows a maximum of $40 \%$ above background at an energy of $0.5 \mathrm{eV}$ above the Fermi level. These values are far from the theoretically predicted 100\% spin-asymmetry at the Fermi level, and we believe that the reason for this discrepancy is the existence of a surface region with a different composition than the bulk [4]. 
The results shown here are of great importance, not only in explaining lower than expected polarization values found in literature, but also because they underscore the importance of characterizing the interface in any effort to understand the properties of spinelectronic junctions. We present clear evidence that changes in surface composition induced by thermal annealing greatly affect the electronic structure of the surface region in $\mathrm{Pb}$-doped manganese perovskite thin films.

[1] H. Kuwahara et al., Science 270 (1995) 961.

[2] R.J. Soulen Jr. et al., Science 282 (1998) 85.

[3] Q.L. Xu et al., Matter. Res. Soc. Symp. Proc. 602 (2000) 75.

[4] C.N. Borca et al., Europhys. Lett. 56 (2001) 722.
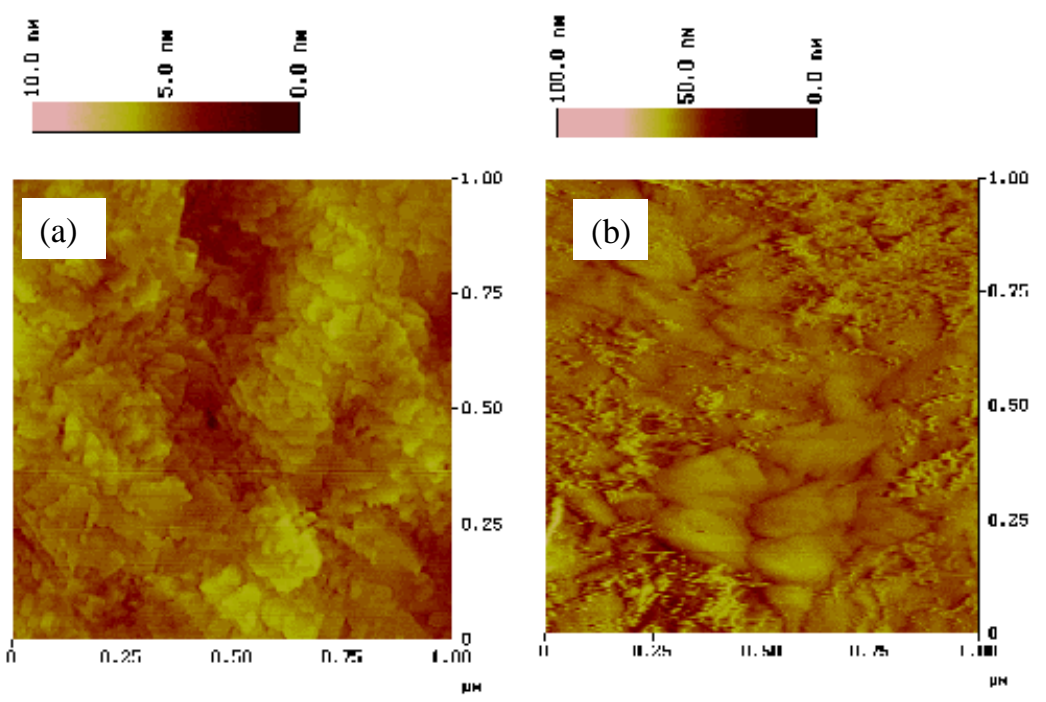

FIG. 1. STM images, $1 \times 1 \mu \mathrm{m}^{2}$ in lateral dimensions, of the $\mathrm{La}_{0.65} \mathrm{~Pb}_{0.35} \mathrm{MnO}_{3}$ thin film 'as-deposited' surface (a) and after heavy annealing treatment (b).
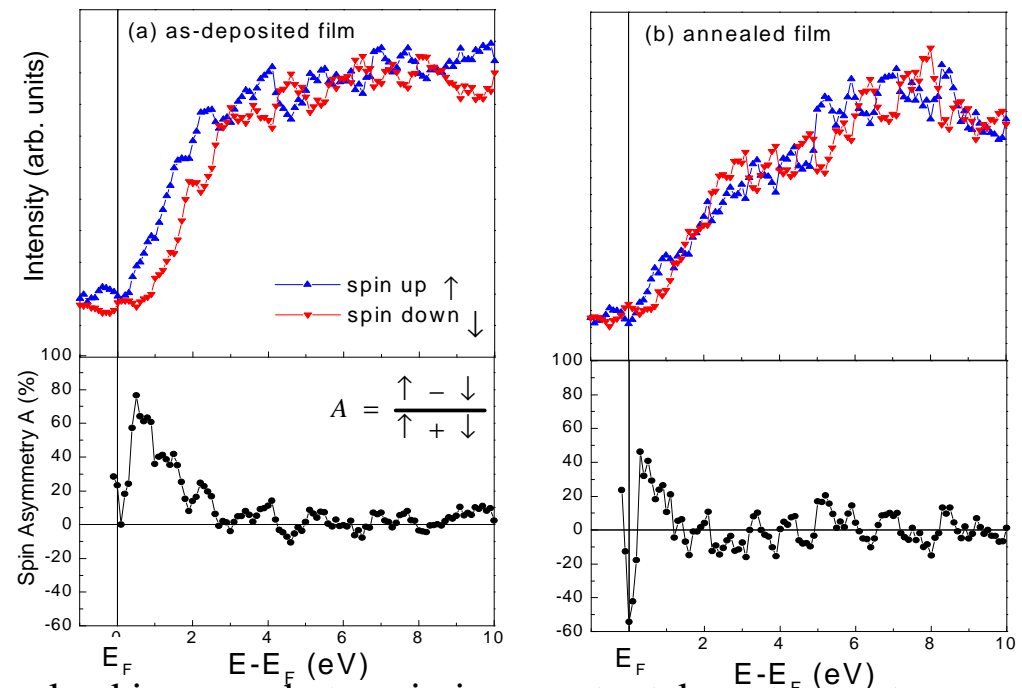

FIG. 2. Spin-resolved inverse photoemission spectra taken at room temperature and normal incidence for the 'as-deposited' $\mathrm{La}_{0.65} \mathrm{~Pb}_{0.35} \mathrm{MnO}_{3}$ surface (a) and for heavily annealed surface (b). Spectra at the bottom of each panel represent the spin asymmetry distributionas a function of incident electron energies. 